\title{
Fast Full-Wave Analysis of Planar Microstrip Circuit Elements in Stratified Media
}

\author{
Rong-Chan Hsieh and Jen-Tsai Kuo, Member, IEEE
}

\begin{abstract}
The fast Hankel transform (FHT) algorithm is implemented in the mixed-potential integral-equation (MPIE) analysis of planar microstrip circuits in stratified media. The spatialdomain Green's functions are accurately and quickly obtained by applying the FHT algorithm to the exact spectral-domain counterparts. Therefore, the entire analysis procedure has high accuracy and efficiency. A nonuniform partition scheme is used to effectively model the rapid change of current distributions around discontinuities. A generalized supplementary equation accounting for arbitrary termination conditions at both feeding and load ends is also derived. The proposed method is used to design a single-stub band-stop filter and a compensated dc block circuit. Experimental measurements are performed to validate the computation.
\end{abstract}

Index Terms-Fast Hankel transform, microstrip discontinuities, mixed-potential integral-equation method.

\section{INTRODUCTION}

D ISCONTINUITIES are inevitable in a microwave integrated circuit (MIC). Their electromagnetic property is a core issue in the design procedure. The discontinuity can be characterized by the spectral-domain approach (SDA) [1], [2] or the spatial-domain technique [3]-[5]. When the geometry of the circuit is complex, no matter which domain is used, the active parts, i.e., conductors in a microstrip-type problem or apertures in a slot-type problem, are divided into subregions for accurately determining the circuit parameters [1]-[5]. Theoretically, the reliability of characterization results will increase if the divisions are made finer. Using the SDA, one has to deal with two-dimensional infinite integrals for setting up the final matrix [1], [2]. The contributions to the integrals from large spectral variables are important if the circuit divisions are small. Therefore, numerical computation could be expensive for circuits with fine geometries. On the other hand, in the spatial-domain technique, only multiple integrals of finite bounds corresponding to extents of each relevant division are required for the computation of matrix entries. Hence, the smaller the divisions, the easier the evaluation of matrix.

The incorporation of the spatial-domain moment method (MM) in the mixed-potential integral-equation (MPIE) formulation is a general and rigorous method for analyzing planar MIC's [3]-[6]. The core of the technique is the calculation of the spatial-domain Green's function, since one has to take

Manuscript received April 22, 1997; revised November 11, 1997. This work was supported by the National Science Council, Taiwan, R.O.C., under Grant NSC 87-2213-E-009-111.

The authors are with the Department of Communication Engineering, National Chiao Tung University, Hsinchu, 300 Taiwan, R.O.C.

Publisher Item Identifier S 0018-9480(98)06148-1. care of the Sommerfeld integral (SI), which involves a highly oscillatory and slowly decaying kernel, the zeroth-order Bessel function of the first kind. Many contributions have been made to this integral [7]-[13]. They fall into two categories. In the first or direct integration approach, the integration path can be the real axis [7] or a deformed path on the complex plane [8]. If the integration is performed along the real axis, singularities of the integrand should be found out and removed via the residue calculus. As for the deformed path integration, the associated Bessel function with complex arguments may cause difficulty in obtaining accurate results.

The second category is an (asymptotic) approximation of the SI. The modal expansion method [11] is a canonical one. The main limitation of this method is that the result converges very slowly in the near-field region. Recently, a new type of approximation called the complex-image method (CIM) has been developed [9]-[13]. Only a few closed-form complex images (images with complex amplitudes and locations) are needed to approximate the SI over a moderate distance range [12]. The two-level approach proposed by Aksun [13] can make the choice of the numbers of complex images and sampling points and the endpoints of the sampling regions robust, so that it provides accurate representation of the Green's function and is much faster compared to the original one-level approximation.

In this paper, the fast Hankel transform (FHT) algorithm [14], [15] is proposed to calculate the spatial-domain Green's function. To the best of authors' knowledge, it is the first time that the FHT algorithm has been employed to calculate the Green's function in a layered dielectric medium. It will be shown that the SI with a smoothed integrand can be reduced to a discrete convolution. The convoluted SI result can be considered as the system response of a digital filter due to the smoothed integrand. An efficient and accurate result can always be achieved, since the filter coefficients associated with the Bessel function have analytic forms and are fast decaying.

The FHT is incorporated in the MPIE technique to characterize the planar microstrip discontinuities. An improved supplementary equation is derived to account for arbitrary load condition at the circuit terminals.

This paper is organized as follows. Section II describes the FHT algorithm and its calculation of the spatial-domain Green's functions. Section III shows the characterization of circuit discontinuities. It includes the partition scheme of the circuit and the derivation of the supplementary equation. In Section IV, the accuracy and efficiency of the FHT algorithm are demonstrated. The proposed method is used to design a 


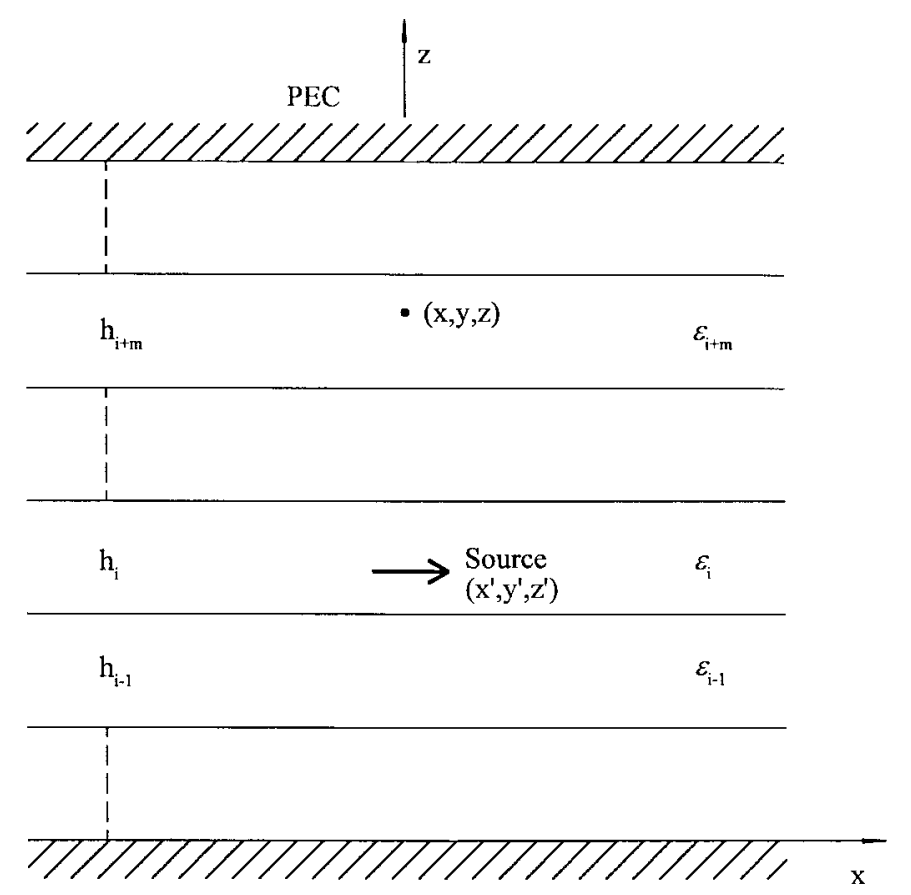

$\mathrm{PEC}$

Fig. 1. A point source embedded in the PEC's bounded multilayered planar structure.

single-stub band-stop filter and a compensated dc block circuit. Experimental measurements are also performed to validate the calculations. Finally, the conclusion is drawn in Section V.

\section{SPATIAL-DOMAIN GREEN's FUnCTIONS}

\section{A. Representations of Spatial-Domain Green's Functions}

In Fig. 1, an infinitesimal electric dipole of unit strength is embedded in the $i$ th layer of a planar dielectric waveguide. The lossless dielectric layers are extended to infinity in both the $x$ and $y$-directions. Two perfect electric conductors (PEC's) are placed at the top and bottom of this structure. The spectraldomain Green's functions for the vector $\left(\tilde{G}_{A}\right)$ and scalar $\left(\tilde{G}_{q}\right)$ potentials can be readily obtained from the generalized reflection and transmission coefficients representations [3]. The spatial-domain counterparts $G_{A, q}$ are the Hankel transforms of $\tilde{G}_{A, q}$

$$
G_{A, q}(\rho)=\frac{1}{4 \pi} \int_{-\infty}^{\infty} \tilde{G}_{A, q}\left(k_{\rho}\right) H_{0}^{(2)}\left(k_{\rho} \rho\right) k_{\rho} d k_{\rho}
$$

where $H_{0}^{(2)}$ is the Hankel function of the second kind of zeroth order. $\tilde{G}_{A, q}$ in (1) have two important features [16]: they have no branch-point singularity since the multilayered structure is covered by two PEC's, and their poles lie on the real and imaginary axes, for the dielectric layers being lossless, and appear as pairs $\left( \pm k_{\rho}\right)$ since $\tilde{G}_{A, q}$ are even functions of $k_{\rho}$.

Before the application of the FHT, the real poles of $\tilde{G}_{A, q}$ must be found out and removed. Let

$$
G_{A, q}(\rho)=G_{A, q}^{e}(\rho)=G_{A, q}^{p}(\rho)
$$

where

$$
\begin{aligned}
G_{A, q}^{e}(\rho)= & \frac{1}{4 \pi} \int_{-\infty}^{\infty} \tilde{G}_{A, q}^{e}\left(k_{\rho}\right) H_{0}^{(2)}\left(k_{\rho} \rho\right) k_{\rho} d k_{\rho} \\
\tilde{G}_{A, q}^{e}\left(k_{\rho}\right)= & \tilde{G}_{A, q}\left(k_{\rho}\right)-\sum_{m} \frac{2 k_{A, q_{\rho m}} \operatorname{Res}_{A, q}\left(k_{A, q_{\rho m}}\right)}{k_{\rho}^{2}-k_{A, q_{\rho m}}^{2}} \\
G_{A, q}^{p}(\rho)= & \frac{1}{4 \pi}(-2 \pi j) \sum_{m} \operatorname{Res}_{A, q}\left(k_{A, q_{\rho m}}\right) \\
& \cdot H_{0}^{(2)}\left(k_{A, q_{\rho m}} \rho\right) k_{A, q_{\rho m}}
\end{aligned}
$$

and $\operatorname{Res}_{A, q}\left(k_{A, q_{\rho m}}\right)$ represent the residues of $\tilde{G}_{A, q}$ at real poles $k_{A, q_{\rho m}}$. In (2), $G_{A, q}^{p}$ are the potentials for propagating surface waves or parallel-plate waveguide modes, which dominate (2) in the far-field region. From the modal expansion method, the potentials $G_{A, q}^{e}$ can be attributed to the contributions from the $\rho$-direction evanescent modes [11]. Since all the $\rho$-direction evanescent modes lie on the negative imaginary axis, $G_{A, q}^{e}$ can be expressed as

$$
\begin{aligned}
G_{A, q}^{e}(\rho)=\frac{1}{4 \pi}(-2 \pi j) \sum_{n=1}^{\infty} \operatorname{Res}_{A, q}\left(-j k_{A, q_{\rho n}}\right) \\
\cdot H_{0}^{(2)}\left(-j k_{A, q_{\rho n}} \rho\right)\left(-j k_{A, q_{\rho n}}\right) .
\end{aligned}
$$

The infinite series in (6) converge very slowly in the near-field region [11], and become inefficient for circuit characterization. However, the imaginary parts of $G_{A, q}^{e}(\rho)$ will be zero, since both $\operatorname{Res}_{A, q}\left(-j k_{A, q_{\rho n}}\right)$ and $H_{0}^{(2)}\left(-j k_{A, q_{\rho n}} \rho\right)$ are purely imaginary if their arguments have zero real parts. Hence, the imaginary parts of $G_{A, q}(\rho)$ are those of $G_{A, q}^{p}(\rho)$.

\section{B. Calculation of Spatial-Domain Green's Functions by the FHT Algorithm}

It is known that (3) can be rewritten as [16]

$$
G_{A, q}^{e}(\rho)=\frac{1}{2 \pi} \int_{0}^{\infty} \tilde{G}_{A, q}^{e}\left(k_{\rho}\right) J_{0}\left(k_{\rho} \rho\right) k_{\rho} d k_{\rho}
$$

where $J_{0}$ is the zeroth-order Bessel function of the first kind. According to [15], (7) can be transformed to a convolution integral

$$
G(v)=\int_{-\infty}^{\infty} F(u) H(v-u) d u
$$

where

$$
\begin{aligned}
F(u) & =\frac{1}{2 \pi} e^{-u} \tilde{G}_{A, q}^{e}\left(e^{-u}\right) \\
G(v) & =e^{v} G_{A, q}^{e}\left(e^{v}\right) \\
H(u) & =e^{u} J_{0}\left(e^{u}\right)
\end{aligned}
$$

with $u=-\ln k_{\rho}$ and $v=\ln \rho$. The convolution integral (8) can be further reduced to the following discrete one:

$$
G^{*}(m \Delta)=\sum_{-\infty}^{\infty} F\left(n \Delta+\Delta_{0}\right) H^{*}\left((m-n) \Delta-\Delta_{0}\right)
$$

where the filter coefficient function $H^{*}(v)$ is defined as

$$
H^{*}(v)=\int_{-\infty}^{\infty} P\left(\frac{u}{\Delta}\right) H(v-u) d u
$$


and $G^{*}$ is the approximation of $G$. The sampling interval $\Delta$ is usually expressed in terms of $N_{\mathrm{DEC}}$, the number of sampling points per decade, as follows:

$$
\Delta=\frac{\ln 10}{N_{\mathrm{DEC}}} .
$$

The sampling shift $\Delta_{0}$ is used to simultaneously shift the sampling points of $F(u)$ and $H^{*}(v)$ in (10). If the interpolation function $P(u)$ is chosen to be [15]

$$
P(u)=\alpha \frac{\sin (\pi u)}{\sinh (\pi \alpha u)}
$$

where $\alpha$ is a smoothness parameter, the function $H^{*}(v)$ has explicit series representations. This filter has two attractive features for numerical calculation: for a given accuracy, an "optimized" filter with minimized length and sampling density $(1 / \Delta)$ can always be achieved by choosing a suitable $\alpha$ value according to the analytical properties of the input function $F(u)$, and the length of the shifted filter is one-third of the nonshifted one if an appropriate $\Delta_{0}$ is introduced so as to sample the filter function $H^{*}(v)$ at its zeros as $v$ becomes large. Therefore, only a limited number of sampling values $F\left(n \Delta+\Delta_{0}\right)$ are needed to obtain accurately converged $G^{*}(m \Delta)$ at each sampling point $\rho_{m}=10^{m / N_{\mathrm{DEC}}}$. The spatialdomain Green's functions all over the interested distance range can be obtained by interpolation.

\section{The Green's Functions with Extended Range}

Now the Green's functions $G_{A, q}(\rho)$ are obtainable for $\rho \in$ $\left[\rho_{\min }, \rho_{\max }\right]$. The value of $\rho_{\max }$ is the maximal dimension of the analyzed circuit, while $\rho_{\min }$ can only reach a small nonzero value. If the source and observation points are located in different dielectric layers, the Green's functions will approach constants as $\rho$ approaches zero. These constants are good approximations of the Green's functions in the region below $\rho_{\min }$. On the other hand, as the source and observation points are located in the same dielectric layer, $G_{A, q}$ can be treated as

$$
G_{A, q}(\rho)=G_{A, q}^{0}(\rho)+G_{A, q}^{1}(\rho) .
$$

The near-field asymptotic expressions for the point source in Fig. 1 are

$$
G_{A}^{0}(\rho)=\frac{\mu_{0}}{4 \pi R_{0}}
$$

and

$$
G_{q}^{0}(\rho)=\frac{1}{4 \pi \varepsilon_{i}}\left(\frac{1}{R_{0}}+\frac{\tilde{R}_{i, j+1}}{R_{1}}+\frac{\tilde{R}_{i, j-1}}{R_{2}}\right)
$$

where

$$
\begin{aligned}
R_{0} & =\sqrt{\rho^{2}+\left(z-z^{\prime}\right)^{2}} \\
R_{1} & =\sqrt{\rho^{2}+\left(2 \sum_{k=1}^{i} h_{k}-z-z^{\prime}\right)^{2}} \\
R_{2} & =\sqrt{\rho^{2}+\left(z+z^{\prime}-2 \sum_{k=1}^{i-1} h_{k}\right)^{2}} \\
\tilde{R}_{i, j} & =\frac{\varepsilon_{i}-\varepsilon_{j}}{\varepsilon_{i}+\varepsilon_{j}}
\end{aligned}
$$

Both (15) and the first term in (16) are direct field potentials from the point source, while the remaining terms in (16) represent the potentials of the images resulting from the adjacent upper and lower dielectric interfaces. The expressions of $G_{A, q}^{0}$ in (15) and (16) are simpler than the quasi-static potentials in the CIM [9]. The reason these simpler expressions are adopted is that the double integrals associated with $G_{A, q}^{0}(\rho)$ can be easily obtained [see (21)]. Note that both $G_{A}^{1}(\rho)$ and $G_{q}^{1}(\rho)$ approach constant values as $\rho$ approaches zero. In the region $\rho<\rho_{\text {min }}$, these constants are good approximations of $G_{A, q}^{1}(\rho)$ and useful for evaluating diagonal elements in the final MM matrix. According to our experience, $\rho_{\min }=\lambda_{0} /(200 \pi)$ is sufficient, with $\lambda_{0}$ being the wavelength in free space.

\section{Characterization OF Circuit Discontinuities}

\section{A. The Nonuniform Partition Scheme and Efficient Matrix Calculation}

In resolving the surface-current distributions of a microstrip discontinuity, the partition scheme is critical for accuracy and efficiency. It is because the currents rapidly change near conductor edges and discontinuities, and it needs fine discretization for accurate determination of circuit parameters. If uniform partition is used, the final MM matrix may become unacceptably large. However, the cylindrical symmetry property of $G_{A, q}$ can greatly reduce the computation effort in evaluating the full MM matrix if the entire circuit is uniformly partitioned [17]. A possible tradeoff is to adopt the partition as follows. In Fig. 2, parallel to the conducting edges, small partitions are first made near these edges and their side dimensions are specified by $d=m \delta$, where $m$ is called the partition parameter and $\delta$ is a small value. In this paper, $\delta=0.1 \mathrm{~mm}$ is adopted. The remaining portions of the circuit are further uniformly discretized to have maximal size less than one-tenth of guided wavelength. Rooftop functions are used to expand the currents in these subregions. Symmetric $\left(J_{x}, J_{y}\right)$ and asymmetric $\left(J_{c x}, J_{c y}\right)$ pairs of basis functions are for adjacent elements with equal and unequal dimensions, respectively. The half-rooftop functions $J_{y L 1}$ and $J_{y L 2}$ are used to model the possibly nonzero currents at the circuit terminals, and $J_{s}$ represents the impressed current. When the Galerkin procedure is used, fourfold integrals associated with the basis functions need computing. These integrals can be reduced to two-dimensional ones [18]. For example,

$$
\begin{aligned}
\left\langle J_{x m^{\prime} n^{\prime}}, G_{A}^{x x} * J_{x m m}\right\rangle & \\
= & \iint_{D_{B^{\prime}}} d x d y J_{x m^{\prime} n^{\prime}}(x, y) \\
& \cdot \iint_{D_{B}} d x^{\prime} d y^{\prime} G_{A}^{x x}\left(x-x^{\prime}, y-y^{\prime}\right) J_{x m n}\left(x^{\prime}, y^{\prime}\right) \\
= & \iint d u d v G_{A}^{x x}(u, v) C F(u, v)
\end{aligned}
$$

where $u=x-x^{\prime}, v=y-y^{\prime}$, and

$$
C F(u, v)=\iint d x d y J_{x m^{\prime} n^{\prime}}(x, y) J_{x m n}(x-u, y-v) .
$$




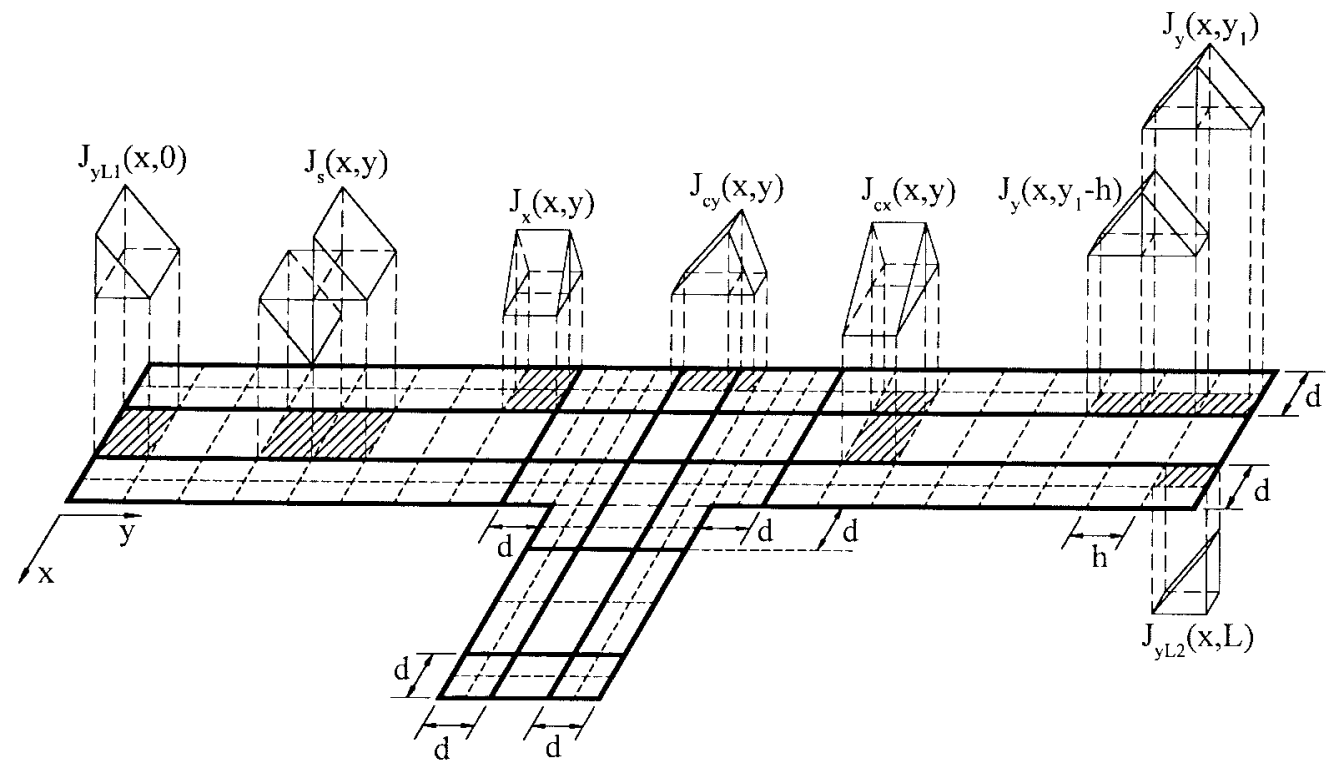

Fig. 2. Nonuniform divisions of the microstrip discontinuity and the rooftop basis functions for expanding the current distribution on the circuit, $d=m \delta, m=2$ here.

According to (14), the reduced twofold integrals (21) can be separated into two parts. The one corresponding to near-field functions $G_{A, q}^{0}(\rho)$ can be analytically derived [19], and the other can be calculated by numerical quadratures [3]. Since the Green's functions have been processed by the FHT, all the matrix elements can be obtained accurately and efficiently.

\section{B. The Improved Supplementary Equation}

Load of arbitrary impedance is often used to model the termination scheme in a practical circuit. Therefore, accurate load modeling is important for any characterization method. In the spatial-domain MM, a supplementary equation is required to deal with the extra load current coefficients for $J_{y L 1}$ and $J_{y L 2}$. Assume that there are only incident and reflected dominant mode waves on the line away from discontinuities. If load $Z_{L}$ is placed at $y=L$, the relation of currents at two positions, e.g., $y=y_{1}$ and $y=y_{1}-h$, can be derived as

$$
J_{y}\left(y_{1}\right)=\frac{1-\Gamma e^{j 2 \beta y_{1}}}{e^{j \beta h}-\Gamma e^{j\left(2 \beta y_{1}-\beta h\right)}} J_{y}\left(y_{1}-h\right)
$$

where

$$
\Gamma=\frac{Z_{L}-Z_{0}}{Z_{L}+Z_{0}} e^{-j 2 \beta L}
$$

and $\beta$ is the phase constant of the line. The supplementary equation in [18] can be obtained by simplifying (23) with $e^{ \pm j \beta h}$ being replaced by $1 \pm j \beta h-\beta^{2} h^{2} / 2$. Hence, (23) is believed to be more general and suitable for accurate circuit characterization.

\section{RESUlTS}

In the near-field region, the real parts of $G_{A, q}(\rho)$ $\left(\operatorname{Re}\left[G_{A, q}(\rho)\right]\right)$ are often several orders of magnitude greater than the imaginary parts $\left(\operatorname{Im}\left[G_{A, q}(\rho)\right]\right)$. However, the accuracy of the whole function is important for use in MM. Note that $\operatorname{Im}\left[G_{A, q}(\rho)\right]$ are $\operatorname{Im}\left[G_{A, q}^{p}(\rho)\right]$ (see Subsection II-A) is automatically confirmed by the FHT algorithm since the integration contour is the real axis and all sample values of $\tilde{G}_{A, q}^{e}\left(k_{\rho}\right)$ are real.

The choice of $\Delta$ and $\Delta_{0}$ is not critical to the accuracy of the FHT algorithm if $\Delta$ is sufficiently small [15]. The size of $\Delta$ is determined by $N_{\mathrm{DEC}}$. The appropriate value of $N_{\text {DEC }}$ can be estimated from $\rho_{\min } / \lambda_{0}, \rho_{\max } / \lambda_{0}$, and the number of poles in the Green's function, since the oscillatory behavior of the Green's function is dominated by these poles in the far-field region. For all the case studies in this paper, $N_{\text {DEC }}=60$ is used. An HP 735 workstation is used to perform the calculations.

The generalized pencil-of-function (GPOF) method [20] is applied to extract the amplitudes and phase constants of the incident and reflected dominant mode waves at circuit terminals. The two-port $S$-parameters of the entire circuit can be calculated from the extracted amplitudes and phase constants [5].

\section{A. Spatial-Domain Green's Functions from the FHT}

Figs. 3 and 4 compare, respectively, $G_{A}^{1}(\rho)$ and $G_{q}^{1}(\rho)$ obtained by the FHT algorithm with those by direct numerical integration. The magnitudes of the full Green's functions $\left|G_{A}(\rho)\right|$ and $\left|G_{q}(\rho)\right|$ are also shown for comparison. Note that $G_{A, q}^{0}\left(=G_{A, q}-G_{A, q}^{1}\right)$ are analytic [see (15) and (16)] and not plotted here. Three poles have been found for this layered structure at $20 \mathrm{GHz}$ : two TM modes at $k_{\rho} / k_{0}=0.7194$ and 1.055 and one TE mode at $k_{\rho} / k_{0}=0.6708$, where $k_{0}$ is the wavenumber in free space. The FHT results (dot points) coincide with the numerical integration results (the solid lines). The total central processing unit (CPU) time used to compute $G_{A, q}\left(\rho_{m}\right)$ at 240 different locations $\left(\rho_{\max } / \rho_{\min }=10^{4}\right)$ for both plots is less than $5 \mathrm{~s}$. The complex constants used to extend the Green's functions to $\rho \leq \rho_{\min }$ can be extracted from the flat parts of $G_{A}^{1}(\rho)$ and $G_{q}^{1}(\rho)$ curves. 


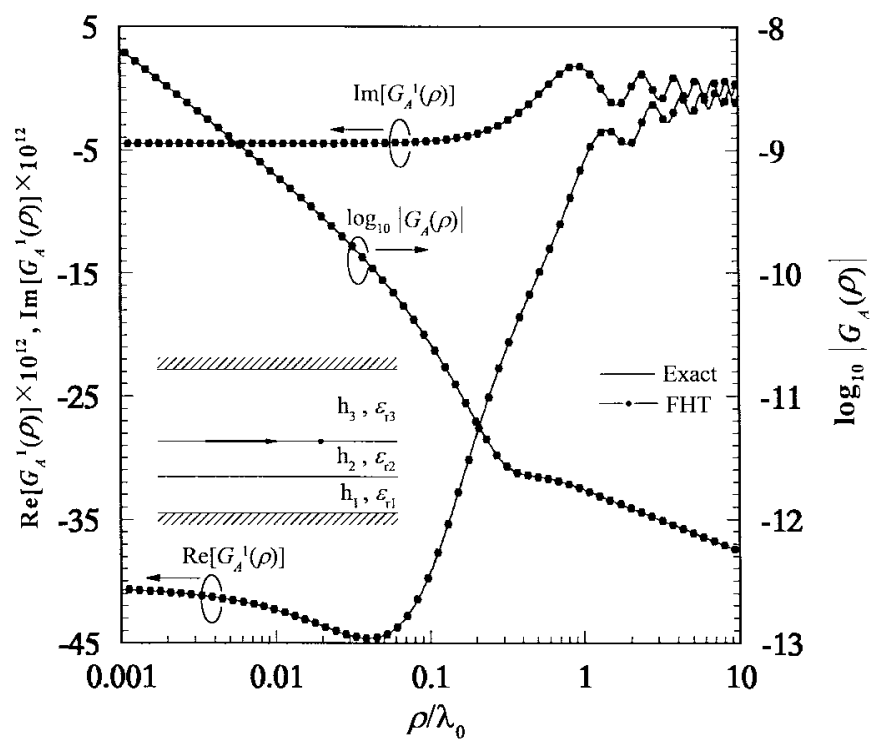

Fig. 3. The real and imaginary parts of $G_{A}^{1}(\rho)$ and the magnitude of $G_{A}(\rho)$ for a horizontal electric dipole in a stratified medium. Parameters: $f=20$ $\mathrm{GHz}, h_{1}=0.508 \mathrm{~mm}, h_{2}=0.254 \mathrm{~mm}, h_{3}=9.238 \mathrm{~mm}, \varepsilon_{r 1}=10.2$, $\varepsilon_{r 2}=2.2$, and $\varepsilon_{r 3}=1.0$.

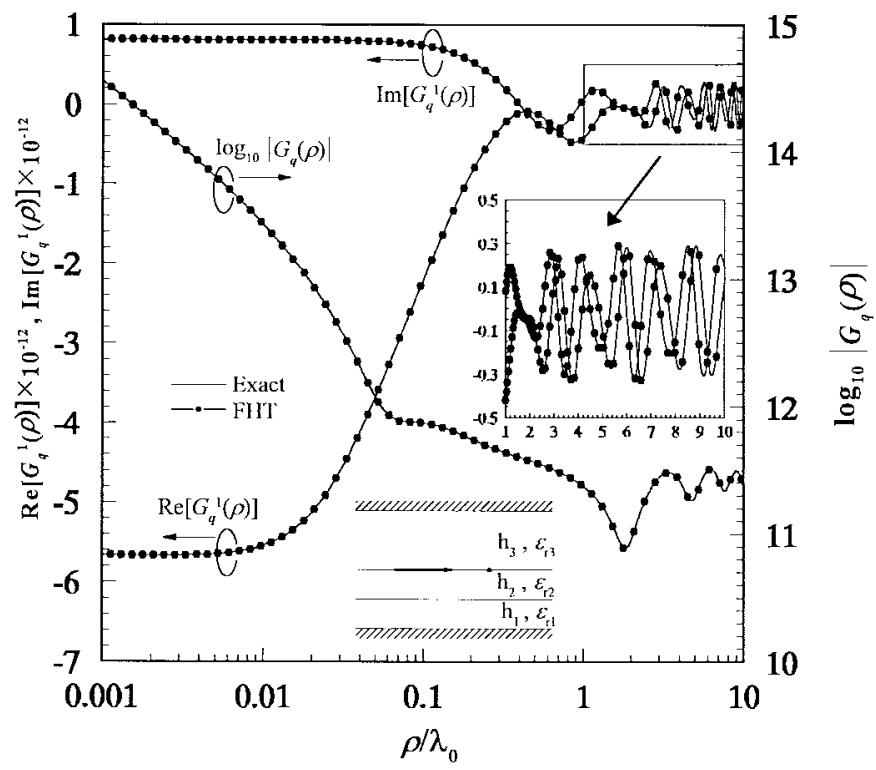

Fig. 4. The real and imaginary parts of $G_{q}^{1}(\rho)$ and the magnitude of $G_{q}(\rho)$ for a horizontal electric dipole in a stratified medium. Parameters and dipole placement are in Fig. 3.

As shown in Fig. 4, the Green's function $G_{q}^{1}(\rho)$ is quickly oscillating in the region $\rho / \lambda_{0} \geq 5$. It is obvious that the number of FHT data points may not be enough to trace the fast varying behavior for linear or simple interpolation. It can be improved by: 1) increasing $N_{\mathrm{DEC}} ; 2$ ) using the far field functions $G_{A, q}^{p}$, which dominate the full Green's functions in this range; 3) enhancing the interpolation technique; or 4) using two separate FHT's with different $N_{\text {DEC }}$ values for different ranges.

Table I compares the real and imaginary parts of $4 \pi \varepsilon_{2} G_{q}(\rho)$ obtained by the FHT algorithm and direct numerical integration at five typical distances. In calculating the convolution
TABLE I

Comparison of the Real and Imaginary Parts of the Scalar Potential Green's Function $\left(4 \pi \varepsilon_{2} G_{q}\right)$ Obtained From the FHT Algorithm AND Exact Numerical Integration at Five Typical Distances. Structure Parameters are in Fig. 3. the Relative Accuracy $\varepsilon=10^{-5}$

\begin{tabular}{c|c|c|c}
\hline & \multicolumn{2}{|c|}{$\operatorname{Re}\left[4 \pi \varepsilon_{2} G_{q}\right]$} & $\operatorname{Im}\left[4 \pi \varepsilon_{2} G_{q}\right]$ \\
\hline$\rho / \lambda_{0}$ & FHT & $\begin{array}{c}\text { Numcrical } \\
\text { Integration }\end{array}$ & FHT \\
\hline $1.017 \times 10^{-3}$ & $8.749562 \times 10^{1}$ & $8.749565 \times 10^{1}$ & $1.985170 \times 10^{-1}$ \\
\hline $1.017 \times 10^{-2}$ & $6.443125 \times 10^{0}$ & $6.443128 \times 10^{0}$ & $1.983287 \times 10^{-1}$ \\
\hline $1.017 \times 10^{-1}$ & $-3.157622 \times 10^{-2}$ & $-3.157593 \times 10^{-2}$ & $1.799537 \times 10^{-1}$ \\
\hline $1.017 \times 10^{0}$ & $-5.197119 \times 10^{-2}$ & $-5.197116 \times 10^{-2}$ & $2.340615 \times 10^{-2}$ \\
\hline $1.017 \times 10^{1}$ & $5.146693 \times 10^{-2}$ & $5.146693 \times 10^{-2}$ & $1.306768 \times 10^{-2}$ \\
\hline
\end{tabular}

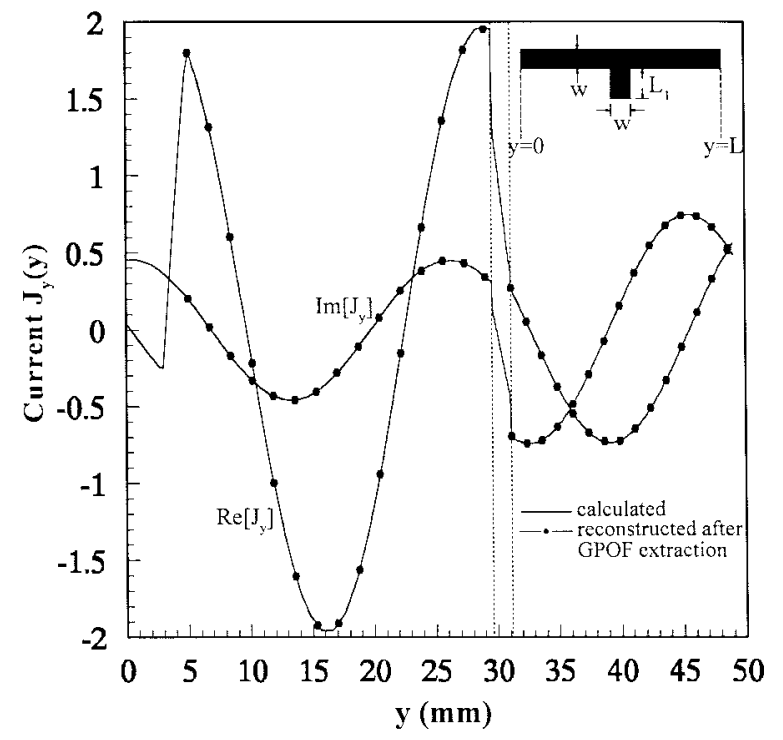

Fig. 5. Calculated (with PEC top cover) and reconstructed current distributions on the main line of a single-stub band-stop filter. Parameters- $f=8.5 \mathrm{GHz}, m=2, w=1.56 \mathrm{~mm}, L_{1}=4.68 \mathrm{~mm}$, $L=49 \mathrm{~mm}, \varepsilon_{r 1}=2.2, \varepsilon_{r 2}=1.0, h_{1}=0.508 \mathrm{~mm}, h_{2}=7.492 \mathrm{~mm}$.

(10), the number of summation terms depends on the relative accuracy $\varepsilon$ [15]. Based on $\varepsilon=10^{-5}$, as shown in Table I, the real-part results have relative deviation less than $10^{-5}$. If $\varepsilon=10^{-9}$ is used, the FHT results agree with those obtained by the direct integration to at least six decimal digits, and the CPU time is increased by $15 \%$. Note that the full complex Green's functions $G_{A, q}$ consist of the FHT results and the residues (5). Although the poles $k_{A, q_{\rho m}}$ in (5) are real, $G_{A, q}^{p}$ are complex in general. It has been shown in Subsection II-A that $\operatorname{Im}\left[G_{A, q}\right]=\operatorname{Im}\left[G_{A, q}^{p}\right]$. The results of $\operatorname{Im}\left[4 \pi \varepsilon_{2} G_{q}(\rho)\right]$ are also listed in Table I for the sake of completeness.

\section{B. Current Distribution of a Microstrip Discontinuity}

In order to demonstrate the validity of the supplementary equation (23), the current distribution on the main line of a single-stub band-stop filter at $8.5 \mathrm{GHz}$ is plotted in Fig. 5 . The partition parameter $m=2$, the structure parameters $w=1.56 \mathrm{~mm}, L_{1}=4.68 \mathrm{~mm}$, and $L=49 \mathrm{~mm}$. The substrate relative dielectric constant $\varepsilon_{r}=2.2$ and the thickness 
TABLE II

Calculated and Measured $S$-Parameters of the Single-Stub Band-Stop Filter at $8.5 \mathrm{GHz}$. Structure Parameters are IN Fig. 5

\begin{tabular}{c|c|c|c|c}
\hline $\begin{array}{c}\text { Partition } \\
\text { Parameter } \mathrm{m}\end{array}$ & $\left|S_{11}\right|$ & $\left|S_{21}\right|$ & $\angle S_{11}$ (degree) & $\angle S_{2 !}$ (degree) \\
\hline 1 & 0.763 & 0.646 & -158.83 & 111.57 \\
\hline 2 & 0.761 & 0.651 & -158.69 & 111.52 \\
\hline 3 & 0.759 & 0.652 & -158.40 & 111.62 \\
\hline 4 & 0.759 & 0.653 & -158.18 & 111.16 \\
\hline Measurement & 0.756 & 0.652 & -159.88 & 110.18 \\
\hline
\end{tabular}

$h_{1}=0.508 \mathrm{~mm}$. The air layer thickness $h_{2}=7.492 \mathrm{~mm}$. The $y$-direction impressed source $J_{s}$ is placed at $y=3.92 \mathrm{~mm}$ and two termination loads are at $y=0$ and $y=49 \mathrm{~mm}$, respectively. The dashed lines at $y=29.6 \mathrm{~mm}$ and $y=31.16$ $\mathrm{mm}$ represent the reference planes of the discontinuity circuit. The rapid current change within these two planes indicates that the major current changes its flow direction from the main line $\left(J_{y}\right)$ to the stub $\left(J_{x}\right)$. The real and imaginary parts of the current distribution for $y>31.16 \mathrm{~mm}$ have identical magnitudes, but a phase difference of $90^{\circ}$, as expected for a traveling wave. The current distribution reconstructed by the GPOF method (dotted line) is also shown in Fig. 5, and it is indistinguishable from the MM result.

\section{Choice of Partition Parameter $m$}

The performance of the nonuniform partition is examined in Table II by comparing the measured $S$-parameters of the single-stub band-stop filter with the calculated results using $m=1,2,3$, and 4 . The deviations of the magnitudes and phase angles of the $S$-parameters for these calculations are within $1 \%$. This reflects the fact that only a small number of fine partitions along the edges of the microstrip lines and around the discontinuities are required for circuit characterization. For the analysis herein, the partition parameter $m=2$.

\section{Measurements}

In the theoretical characterizations of the microstrip discontinuities in this paper, a PEC cover is always placed $7.492 \mathrm{~mm}$ above the circuits. Fig. 6 plots the calculated and measured $S$-parameters of the single-stub band-stop filter in Fig. 5. From the theoretical and measured (with PEC top cover) results, it indicates that the center frequency is accurately predicted and both frequency responses agree well. As the top cover is removed from the circuit, the center frequency is lowered about $90 \mathrm{MHz}$.

In Fig. 7, the response of a dc block circuit with a compensation overlap coupled patch is investigated. The center frequency is designed at $f_{0}=11.5 \mathrm{GHz}$. The gap $G$ and the length of the overlap patch $L_{1}$ are 0.5 and $8.5 \mathrm{~mm}$, respectively. The strip width $w=1.56 \mathrm{~mm}$ and the circuit longitudinal size $L=71.2 \mathrm{~mm}$. The dielectric layers underneath the gap have $\varepsilon_{r 1}=\varepsilon_{r 2}=2.2$ and $h_{1}=h_{2}=0.254 \mathrm{~mm}$. The air layer thickness $h_{3}$ is $7.492 \mathrm{~mm}$. As expected, the circuit has good insertion loss $\left(\left|S_{21}\right|\right)$ at the center frequency. For the frequencies away from $f_{0}$, the predictions by the MPIE

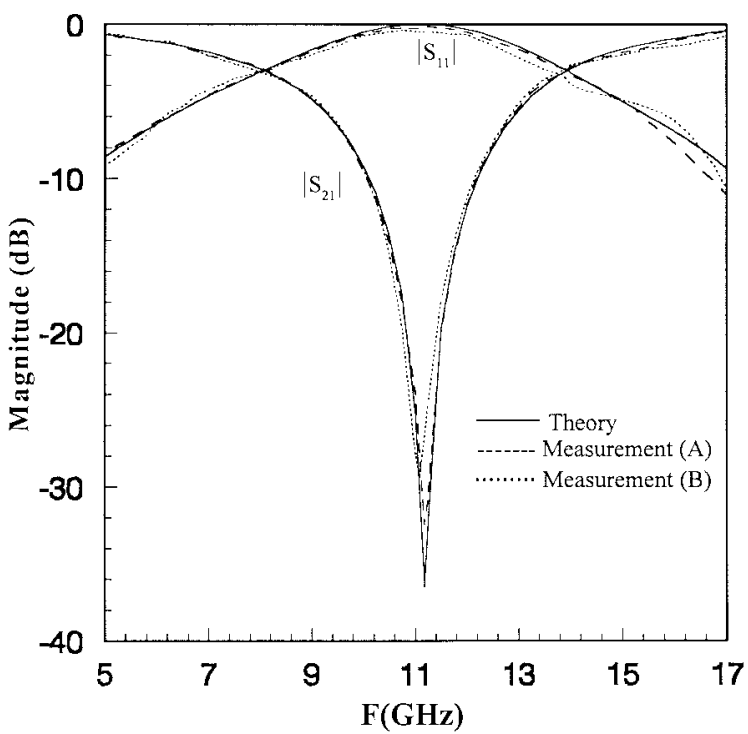

Fig. 6. The theoretical (with PEC top cover) and experimental $S$-parameters of the single-stub band-stop filter in Fig. 5. (- - - )-Measurement $(A)$ with PEC top cover. $(\cdots \cdots)-$ Measurement $(B)$ without PEC top cover.

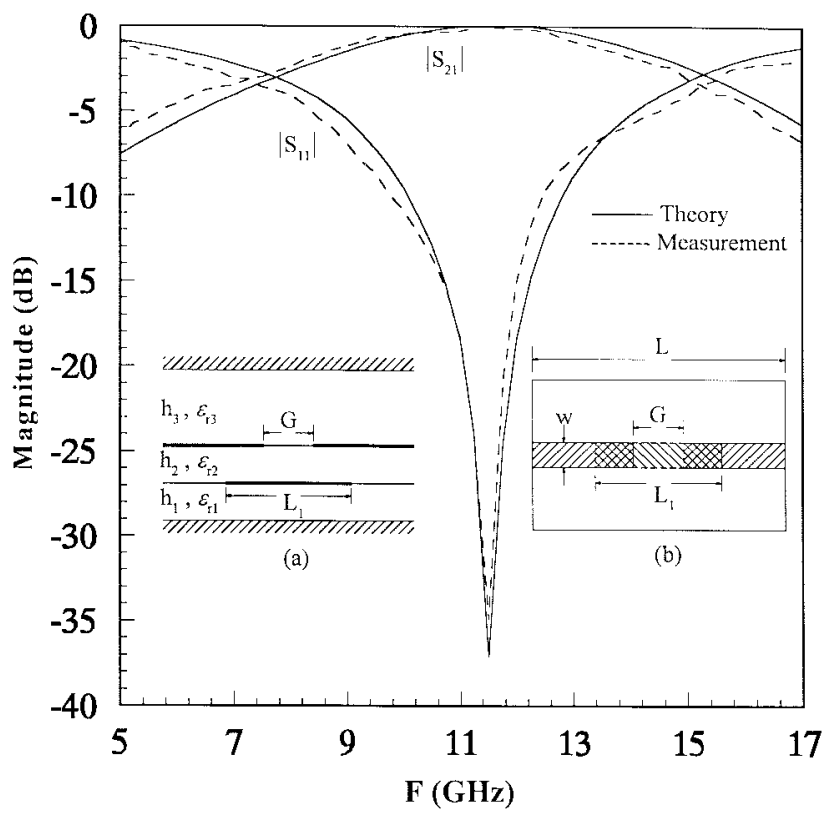

Fig. 7. The theoretical and experimental $S$-parameters of the compensated dc block circuit. Inset-(a) side view, $h_{1}=h_{2}=0.254 \mathrm{~mm}$, $h_{3}=7.492 \mathrm{~mm}, \varepsilon_{r 1}=\varepsilon_{r 2}=2.2, \varepsilon_{r 3}=1.0$. Inset-(b) top view, $G=0.5 \mathrm{~mm}, L_{1}=8.5 \mathrm{~mm}, L=71.2 \mathrm{~mm}, w=1.56 \mathrm{~mm}$.

with FHT have good agreement with the measurements. In the measurements, the top cover has negligible influences on this circuit.

\section{CONCLUSION}

The FHT algorithm is proposed to deal with the highly oscillatory and slowly decaying SI's for calculating the spatialdomain Green's functions. Both the real and imaginary parts of the Green's functions can be accurately and efficiently calculated over a broad distance range $\left(\rho_{\max } / \lambda_{0}=10\right)$. 
A generalized supplementary equation to account for the possibly nonzero currents at circuit terminals is derived. In the nonuniform partition scheme, for the particular case study in Subsection IV-C, the characterized circuit parameters are found insensitive to the value of partition parameter. From the good agreement of the theoretical and experimental $S$-parameters, the validity of the analysis procedure is readily confirmed. It is obvious that the FHT algorithm is well suitable, in conjunction with the MPIE formulation, for efficient and accurate analysis of arbitrary planar-microstripdiscontinuity geometries embedded in stratified media.

\section{ACKNOWLEDGMENT}

The authors thank Prof. N. B. Christensen at the University of Aarhus, Aarhus, Denmark, for his FHT code. Thanks are also due to the anonymous reviewers for their help in enhancing the quality of this paper.

\section{REFERENCES}

[1] R. H. Jansen and W. Wertgen, "A 3D field-theoretical simulation tool for the CAD of mm-wave MMIC's," Alta Freq., vol. LVII, no. 5, pp. 203-216, June 1988.

[2] C.-Y. Lee, Y. Liu, and T. Itoh, "The effects of the coupled slotline mode and air-bridges on CPW and NLC waveguide discontinuities," IEEE Trans. Microwave Theory Tech., vol. 43, pp. 2759-2765, Dec. 1995.

[3] M.-J. Tsai, F. D. Flaviis, O. Fordham, and N. G. Alexopoulos, "Modeling planar arbitrarily shaped microstrip elements in multilayered media," IEEE Trans. Microwave Theory Tech., vol. 45, pp. 330-337, Mar. 1997.

[4] D. C. Chang and J. X. Zheng, "Electromagnetic modeling of passive circuit elements in MMIC," IEEE Trans. Microwave Theory Tech., vol. 40, pp. 1741-1747, Sept. 1992.

[5] I. Park, R. Mittra, and M. I. Aksun, "Numerically efficient analysis of planar microstrip configurations using closed-form Green's functions," IEEE Trans. Microwave Theory Tech., vol. 43, pp. 394-400, Feb. 1995.

[6] J. R. Mosig, "Arbitrarily shaped microstrip structures and their analysis with a mixed potential integral equation," IEEE Trans. Microwave Theory Tech., vol. 36, pp. 314-323, Feb. 1988

[7] P. B. Katehi and N. G. Alexopoulos, "Real axis integration of Sommerfeld integrals with applications to printed circuit antennas," J. Math. Phys., vol. 24, pp. 527-533, Mar. 1983.

[8] Y. Rahmat-Samii, R. Mittra, and P. Parhami, "Evaluation of Sommerfeld integrals for lossy half-space problems," Electromagnetics, vol. 1, pp. 1-28, Jan. 1981

[9] D. G. Fang, J. J. Yang, and G. Y. Delisle, "Discrete image theory for horizontal electric dipoles in a multilayered medium," Proc. Inst. Elect. Eng., vol. 135, pt. H, pp. 297-303, Oct. 1988.

[10] Y. L. Chow, J. J. Yang, D. G. Fang, and G. E. Howard, "A closed-form spatial Green's function for the thick microstrip substrate," IEEE Trans. Microwave Theory Tech., vol. 39, pp. 588-592, Mar. 1991.

[11] J. J. Yang, Y. L. Chow, G. E. Howard, and D. G. Fang, "Complex images of an electric dipole in homogeneous and layered dielectrics between two ground planes," IEEE Trans. Microwave Theory Tech., vol. 40, pp. 595-600, Mar. 1992.
[12] M. I. Aksun and R. Mittra, "Derivation of closed-form Green's functions for a general microstrip geometry," IEEE Trans. Microwave Theory Tech., vol. 40, pp. 2055-2062, Nov. 1992.

[13] M. I. Aksun, "A robust approach for the derivation of closed-form Green's functions," IEEE Trans. Microwave Theory Tech., vol. 44, pp. 651-658, May 1996.

[14] H. K. Johansen and K. Sorensen, "Fast Hankel transforms," Geophys. Prospecting, vol. 27, pp. 876-901, 1979.

[15] N. B. Christensen, "Optimized fast Hankel transform filters," Geophys. Prospecting, vol. 38, pp. 545-568, 1990.

[16] W. C. Chew, Waves and Fields in Inhomogeneous Media. Piscataway, NJ: IEEE Press, 1995.

[17] R. C. Hsieh and J. T. Kuo, "Fast full-wave characterization of arbitrary planar microstrip geometries," in Asia-Pacific Microwave Conf., Hong Kong, Dec. 1997, pp. 685-688.

[18] M. I. Aksun and R. Mittra, "Estimation of spurious radiation from microstrip etches using closed-form Green's functions," IEEE Trans. Microwave Theory Tech., vol. 40, pp. 2063-2069, Nov. 1992.

[19] L. Alatan, M. I. Aksun, K. Mahadevan, and M. T. Birand, "Analytical evaluation of the MoM matrix elements," IEEE Trans. Microwave Theory Tech., vol. 44, pp. 519-525, Apr. 1996.

[20] Y. Hua and T. K. Sarkar, "Generalized pencil-of-function method for extracting poles of an EM system from its transient response," IEEE Trans. Antennas Propagat., vol. 37, pp. 229-234, Feb. 1989.

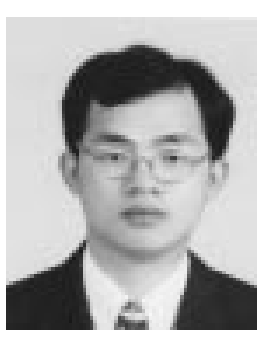

Rong-Chan Hsieh was born in Taiwan, R.O.C., on February 3, 1966. He received the B.S. degree in physics from the National Changhua University of Education, Taiwan, R.O.C., in 1988, the M.S degree in electrophysics from the National Chiao Tung University (NCTU), Taiwan, R.O.C., in 1990, and is currently working toward the Ph.D. degree.

From 1992 to 1993, he was with the EMC Testing Department, Electronics Testing Center, Taiwan, R.O.C.. His research interests include the analysis and design of microwave and millimeter-wave circuits.

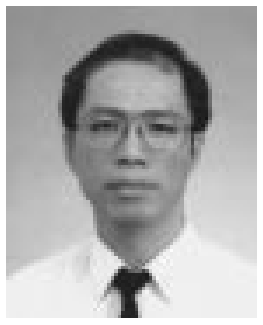

Jen-Tsai Kuo (S'88-M'92) received the B.S. degree in communication engineering from the National Chiao Tung University (NCTU), Taiwan, R.O.C., in 1981, the M.S. degree in electrical engineering from the National Taiwan University, Taiwan, R.O.C., in 1984, and the Ph.D. degree from the Institute of Electronics, NCTU, in 1992.

Since 1984, he has been with the Department of Communication Engineering, NCTU, as a Lecturer in both the Microwave and Communication Electronics Laboratories. From 1995 to 1996, he was a Visiting Scholar at the University of California at Los Angeles. His research interests include the analysis and design of high-frequency electronics and microwave circuits, high-speed interconnects and packages, field-theoretical studies of guided waves, and numerical techniques in electromagnetics. 